\title{
Les médiations institutionnelles de la confiance en entreprise. Une comparaison franco-allemande
}

Institutional Mediations Fostering Trust Within Companies. A Franco-German Comparison

\section{Axel Pohn-Weidinger et Bénédicte Zimmermann}

\section{(2) OpenEdition}

Journals

Édition électronique

URL : https://journals.openedition.org/travailemploi/9748

DOI : 10.4000/travailemploi.9748

ISSN : 1775-416X

Éditeur

DARES - Ministère du Travail

Édition imprimée

Date de publication : 1 mars 2020

Pagination : $5-30$

ISSN : 0224-4365

Référence électronique

Axel Pohn-Weidinger et Bénédicte Zimmermann, « Les médiations institutionnelles de la confiance en entreprise. Une comparaison franco-allemande », Travail et Emploi [En ligne], 161 | 2020, mis en ligne le 01 mars 2021, consulté le 28 septembre 2021. URL : http://journals.openedition.org/travailemploi/ 9748 ; DOI : https://doi.org/10.4000/travailemploi.9748 


\title{
Les médiations institutionnelles de la confiance en entreprise. Une comparaison franco-allemande*
}

\author{
Axel Pohn-Weidinger ${ }^{* *}$, Bénédicte Zimmermann ${ }^{* * *}$
}

\begin{abstract}
Les dispositifs de formation professionnelle et de représentation des salariés sont associés dans les recherches comparatives franco-allemandes à des arrangements institutionnels qui favoriseraient davantage la confiance des salariés allemands que des salariés français dans l'entreprise qui les emploie. Le propos de l'article est de soumettre cette thèse à une enquête compréhensive au sein de deux multinationales qui traversent un moment d'instabilité organisationnelle. Il rend compte de la transformation et de l'hybridation de ces arrangements institutionnels, tout en montrant l'impact qu' ont sur ces derniers les contingences organisationnelles liées à l'histoire des sites enquêtés et à leurs positions respectives au sein de la multinationale.
\end{abstract}

$\mathrm{L}$ a question de la confiance fait aujourd'hui l'objet d'un intérêt renouvelé dans un monde du travail en proie à de profondes incertitudes alimentées par des transformations tous azimuts: révolution digitale, économie globalisée et financiarisée, entreprises flexibilisées, remise en question des arrangements sociaux construits autour de l'emploi stable et à plein temps, etc. Ces mutations contribuent à distendre les cadres normatifs de l'action pour faire davantage place à la responsabilité et à l'initiative individuelles (LALLEMENT, ZimMERMAnN, 2019). Au niveau de l'entreprise, la norme de l'action prescrite et prévisible, principe du taylorisme, est détrônée par celle de l'action qui convient (THÉVENOT, 1990), au sens où elle est appropriée à la situation et ne peut être définie par avance. Au niveau des politiques publiques, la sécurité de l'emploi, qui désigne un état stable indépendant de la personne, fait place à la sécurisation des parcours, qui renvoie à un processus en permanente évolution, lié aux propriétés singulières des personnes.

\footnotetext{
* Nous remercions Jürgen Kädtler et Léa Renard pour leur lecture et leurs commentaires d'une première version de ce texte.

** Université de Strasbourg et Centre Georg Simmel (EHESS); apohnweidinger@unistra.fr.

*** EHESS (Centre Georg Simmel), Paris et Wissenschaftskolleg, Berlin; benedicte.zimmermann@ehess.fr.
} 
Comme l'a montré Niklas LuHMANN (2006), lorsque les routines et les acquis sont remis en question, lorsque surgit une interrogation quant au futur et à la manière appropriée d'agir, se pose la question de la confiance. À la suite de Georg SimmeL (1999 [1908], p. 356) qui définit la confiance comme une « hypothèse sur une conduite future assez sûre pour qu'on fonde sur elle l'action pratique », N. LuHMANN (2006) fait de la confiance un «mécanisme de réduction de la complexité sociale » qui permet d'agir dans le présent, de se coordonner avec autrui et de se projeter dans le futur. Nous retenons de ces auteurs que la confiance revêt une dimension tout à la fois pratique, cognitive et évaluative. Nous la définissons à ce stade comme une conjecture positive qui procède d'un jugement pratique quant à la clôture d'une situation ou d'une action qui est tributaire de l'agissement d' autrui. Elle signifie une forme de pouvoir donné à autrui, tout en impliquant une attente de réciprocité. À ce titre, elle constitue une dimension structurante de la capacité d'agir de son émetteur aussi bien que de son destinataire.

En braquant les projecteurs sur la pluralité des régimes d'action et l'activité de coordination qui en résulte, l'économie des conventions a contribué à alimenter une riche veine de travaux sur le rôle de la confiance dans les échanges économiques (EYMARD-DUVERNAY, 1989; KARPIK, 1996; LAZARIC, LORENZ, 1998; LIVET, REYNAUD, 1998). Nous en retenons que la confiance facilite la coopération entre des acteurs qui ne se connaissent pas forcément et se trouvent dans une asymétrie de pouvoir et d'information. Cette asymétrie caractérise tout particulièrement la relation contractuelle entre l'employeur et le salarié individuel, que l'organisation collective des relations professionnelles vise à rééquilibrer.

C'est à travers la comparaison franco-allemande que nous appréhendons ici la question de la confiance dans l'entreprise. Lorsque Francis FUKUYAMA (1995) soutient qu'il existe un lien causal entre le niveau de confiance qui règne au sein d'une société et sa performance économique, du fait des coûts de transaction que la confiance permettrait de réduire, il fait de la France l' archétype d'une société à faible confiance, et donc à faible performance, par contraste avec l'Allemagne qui serait son envers. D'autres travaux ont confirmé une différence de niveau de la confiance observable dans les deux pays, mais en en complexifiant l'analyse (UsUNIER, 2000a; WILLINGER et al, 2001). Jean-Claude UsUNIER (2000b, p. 223 et 224) identifie ainsi une plus grande propension des Allemands à faire confiance dans une relation impersonnelle, là où les Français seraient plus enclins à la confiance spontanée dans une relation interpersonnelle (ibid., p. 225). Au-delà de la culture nationale invoquée par F. FuKUYAMA, il pointe le rôle des arrangements institutionnels dans la production de ces différences.

Les travaux pionniers de Marc MAURICE, François SEllier et Jean-Jacques SiLVESTRE (1982) ont quant à eux fait des systèmes éducatifs et de relations professionnelles des facteurs décisifs de différenciation du rapport salarial en France et en Allemagne. Le premier ensemble d'institutions forge un type particulier de « rapport éducatif », le second de « rapport industriel » (au sens de relations professionnelles). Ces auteurs relèvent en outre le rôle tout aussi déterminant d'un troisième rapport qu'ils qualifient d' « organisationnel » et qui se rapporte à la structure des emplois, à la 
hiérarchie des acteurs dans l'entreprise, aux formes d'encadrement et de coopération. Le pont que construisent ces auteurs entre les systèmes institutionnels, organisationnels et de relations professionnelles, pour montrer comment leurs interdépendances forgent des configurations sociétales spécifiques, reste une contribution essentielle à la comparaison franco-allemande, qui mérite d'être interrogée sous l'angle de la production de la confiance en entreprise. Explorer la production de la confiance au travail suppose cependant de franchir un pas supplémentaire et de pousser la porte des entreprises pour y mener l'enquête au plus près des contextes de travail, plutôt que de s'en tenir à des données générales sur les entreprises françaises et allemandes considérées comme deux groupes homogènes. Notre démarche se situe à cet égard au croisement entre l'approche sociétale et l'approche comparée des organisations telle qu'elle a par exemple été développée par Mathilde BOURRIER (1991) dans sa comparaison du fonctionnement de quatre centrales nucléaires en France et aux États-Unis. Fort différentes, ces veines de recherche ont toutes deux essuyé les critiques de Philippe D'IRIBARNE $(1991,2005)$ qui leur reproche de s'affranchir de la notion de culture nationale dans l'explication des différences. Pour ce qui nous concerne, nous considérons que les institutions nationales qui encadrent le travail, son marché et son organisation sont constitutives de cultures nationales en ce qu'elles façonnent les cadres de l'agir situé. C'est afin d'étudier cet « encastrement social des multinationales » (HEIDENREICH, 2012) que nous estimons particulièrement important de croiser les échelles institutionnelles, organisationnelles et individuelles. À la suite d'Ann SwIDLER (1986), nous appréhendons la culture en action, c'est-à-dire comme point d'appui mobilisé dans l'agir situé, plutôt que comme un ensemble de valeurs abstraites partagées dont on saurait par avance comment elles impactent l'agir.

L'objet de notre propos n'est pas de mesurer la confiance ou d'en établir un benchmark, mais de soumettre les médiations institutionnelles de la confiance identifiées par les auteurs des années 1980-1990 à l'épreuve de leurs expressions concrètes dans l'entreprise aujourd'hui. Les systèmes éducatifs et de relations professionnelles favoriseraient tous deux davantage la confiance intra-organisationnelle en Allemagne qu'en France; le premier par l'intégration précoce dans l'entreprise que permet de développer le système dual de formation professionnelle en alternance; le second par un « partenariat conflictuel » (Konfliktpartnerschaft) entre employeurs et représentants des salariés (MÜLLER-JENTSCH, 1999). Ces médiations sont de nature institutionnelle au sens où elles font l'objet d'une prescription et d'une codification par les politiques publiques, qui s'imposent aux organisations, même si ces dernières bénéficient d'une importante latitude de mise en œuvre.

L'analyse s'adosse à une enquête comparative franco-allemande menée dans le cadre du projet ANR/DFG Devent consacré à l'étude de la capacité d'agir des salariés dans les entreprises françaises et allemandes (encadré 1). Elle se concentre sur deux d'entre elles, l'entreprise OilGum dans la chimie et l'entreprise Flybest dans l'aéronautique, qui se sont révélées les cas les plus contrastés du point de vue de la problématique de la confiance; OilGum confirmant à grands traits les conclusions des travaux comparatifs franco-allemands, Flybest les invalidant (encadré 2). 


\section{ENCADRÉ 1}

\section{Terrain et méthode}

\section{L'enquête Devent (Développement professionnel et capacité d'agir des salariés en entreprise). La France et l'Allemagne au prisme des multinationales}

Déployée entre 2014 et 2018, l'enquête Devent a été financée à titre principal par l'Agence nationale de la recherche (ANR) et la Deutsche Forschungsgemeinschaft (DFG), avec une contribution du Trust Management Institute (TMI) et de l'entreprise Air Liquide. Elle a été mise en œuvre par une équipe franco-allemande associant des chercheurs du Centre Georg Simmel (UMR 8131 CNRS/EHESS) et du Soziologisches Forschungsinstitut (SOFI) de Göttingen.

L'enquête a porté sur six multinationales des secteurs de la chimie, de la pharmacie et de la métallurgie. Pour chacune, nous avons étudié deux unités comparables du point de vue de leur activité, l'une en France, l'autre en Allemagne.

Qualitative, la méthode combine et confronte différentes sources. Au cours d'un séjour de deux semaines sur chaque site, un binôme franco-allemand de chercheurs a procédé par observations et par entretiens auprès de différentes catégories du personnel.

Une trentaine d'entretiens d'une durée moyenne de 90 minutes ont été réalisés pendant le temps de travail sur chaque site avec des salariés en contrat à durée indéterminée (CDI) avec plus d'un an d'ancienneté, de différents niveaux de qualifications et aux fonctions variées, incluant des représentants du personnel. Le choix de limiter l'enquête au noyau stable des salariés en CDI avait pour objectif de réduire la complexité, déjà grande, d'une analyse comparée des multiples facteurs qui impactent la capacité d'agir. En parallèle ont été menés des entretiens avec cinq à dix personnes des services du personnel de chaque groupe multinational. L'ensemble des entretiens a été transcrit et analysé à l'aide du logiciel NVivo.

Les enquêtes ont fait l'objet d'un accord de confidentialité, raison pour laquelle les noms des groupes et des personnes qui y ont participé ont été pseudonymisés.

Pour chaque multinationale, un site français et un site allemand d'activité similaire - la production de harnais électriques pour Flybest, de caoutchouc pour OilGum ont fait l'objet d'une investigation. Les deux entreprises ont pour point commun de traverser un moment d'instabilité organisationnelle: pour Flybest, en raison d'une réorganisation du travail liée à une importante baisse d'activité sur une des lignes et une forte augmentation sur d'autres; pour OilGum, en raison de la formation d'un joint venture avec une entreprise pétrolière étrangère. Les deux situations sont de nature à mettre à l'épreuve la confiance des salariés dans leur entreprise.

L'enquête s'est attachée à questionner la confiance à différentes échelles (qui interagissent les unes avec les autres) dans l'entreprise : la confiance interpersonnelle entre membres d'une même équipe, entre le salarié et son manager direct; la confiance entre équipes, divisions, ou domaines de spécialités ; la confiance des salariés dans la direction de site, de l'usine et de la multinationale, plus largement dans l'entreprise considérée comme entité; enfin, leur confiance dans les instances de représentation du personnel. 


\section{ENCADRÉ 2}

\section{OilGum et Flybest}

\section{OilGum (enquête: septembre-novembre 2016)}

Producteur de caoutchouc synthétique, OilGum est un joint venture créé par la multinationale TopGum et l'entreprise pétrolière BigOil en 2016. TopGum, qui compte environ 16000 salariés, transfère à cette occasion ses activités de production de caoutchouc à OilGum. Les unités sur lesquelles a porté l'enquête comptent près de 300 salariés permanents en France, 500 en Allemagne. Tandis que l'unité française se trouve dans une zone à faible densité d'industries chimiques, l'unité allemande se situe à l'intérieur d'un Chempark (zone industrielle dédiée à la chimie) où sont employés environ 10500 salariés. Historiquement, l'ensemble des enseignes présentes appartenait à une seule et même entreprise chimique allemande (que nous appellerons Hoffmann) qui, à partir des années 1990, a vendu ses différentes composantes ou les a transformées en sociétés autonomes.

Les personnes interviewées sur le site allemand (OilGum DE) travaillent dans deux ateliers qui comptent respectivement 300 et 50 salariés répartis sur plusieurs lignes de production. En France (OilGum FR), l'enquête s'est concentrée sur l'atelier qui fabrique le même type de produit, selon le même procédé, que les deux ateliers allemands. Quatrevingts salariés y travaillent sur plusieurs lignes. Chez OilGum DE, la polyvalence est très développée, la plupart des ouvriers connaissent l'ensemble des postes et sont rémunérés de façon identique, alors que chez OilGum FR, les postes, tâches et rémunérations sont plus finement différenciés. Selon une logique de polyvalence décroissante, le contremaître y encadre le superviseur, le conducteur de ligne, l'opérateur polyvalent et l'opérateur.

\section{Flybest (enquête: juin-juillet 2016)}

Constructeur aéronautique, le groupe Flybest compte en 2016 près de 50000 salariés en France et autant en Allemagne. Les deux unités de production de câblages électriques sur lesquelles a porté l'enquête comptent respectivement 250 salariés permanents pour le site français (auxquels il faut rajouter $0,4 \%$ à $2 \%$ de travailleurs intérimaires selon les périodes ; 0,8 \% au moment de l'enquête) et 125 salariés permanents pour le site allemand (auxquels il faut rajouter $12 \%$ à $16 \%$ de travailleurs intérimaires; $12 \%$ au moment de l'enquête). L'assemblage de harnais, la fabrication de boîtiers et de coffres électriques, le dépannage sur les chaînes d'assemblage final des avions font partie des activités des unités.

Sur le site français (Flybest FR), le travail est organisé en cinq lignes, chacune dédiée à un modèle d'avion et toutes réunies dans un grand hangar. Sur le site allemand (Flybest DE), le travail est organisé en équipes qui se distribuent sur les trois étages d'un bâtiment industriel. L'unité allemande accueille en outre au sein de l'équipe dédiée la production des boîtiers électriques des ouvriers en capacité de travail réduite provenant d'autres unités. C'est là son originalité par rapport à l'unité française. Si la structure hiérarchique est plus plate en Allemagne avec des porte-paroles d'équipes élus plutôt que des leaders nommés par la direction, Flybest FR, prenant inspiration du modèle de l' « entreprise libérée ${ }^{1}$ », s'engage aussi au moment de l'enquête vers la suppression de l'échelon hiérarchique de proximité. Dans les deux cas sont déployés des dispositifs de management participatif.

1. L'entreprise libérée est un modèle managérial qui préconise des hiérarchies plates et la suppression de l'encadrement de proximité afin de « libérer » l'initiative et de responsabiliser les salariés (CARNEY, GETZ, 2013 [2009]). 
Le présent article se concentre sur les deux derniers niveaux: la confiance systémique $^{1}$ des salariés dans l'entreprise comme entité et celle envers les instances de représentation. De façon inattendue, dans les deux entreprises étudiées, c'est au sein des unités allemandes plutôt que françaises que nous observons que la confiance des salariés, à ces deux niveaux, se trouve malmenée.

Une première partie consacrée aux parcours d'entrée et d'installation dans l'entreprise, une deuxième aux instances et dispositifs de représentation des salariés montrent que ces deux dimensions classiques de la comparaison franco-allemande restent en partie seulement pertinentes. Les résultats vont dans le sens d'une hybridation des systèmes de formation professionnelle français et allemands identifiée par Éric VERDIER (2017) et révèlent les défis auxquels se trouve confronté le système allemand de codétermination. Une troisième partie suggère que l'impact de ces deux types d'arrangements institutionnels sur la confiance des salariés dans l'entreprise est en outre fortement tributaire des configurations et des contingences organisationnelles liées à la position de chacune des unités dans les rapports de force au sein de la multinationale.

\section{Au-delà de la formation professionnelle initiale, les parcours d'installation dans l'entreprise}

Dans leur enquête comparée sur la production de la confiance entre partenaires du système de formation professionnelle initiale en France et en Allemagne, Éric DAVOINE, Björn WALLISER et Jean-Charles RIERA (2000) identifient deux principaux ressorts de la confiance: l'un de nature contractuelle, l'autre de nature tacite. La confiance contractuelle s'appuie sur un socle de normes juridiques qui, en facilitant la « contrôlabilité » des conduites des acteurs impliqués, permettent d'instaurer une relation de confiance entre des personnes qui ne se connaissent pas (PARADEISE, PORCHER, 1990). La confiance tacite repose, quant à elle, sur des relations de proximité. Elle s'alimente à trois canaux : réputationnel (lié aux signaux diffusés par les partenaires), relationnel (lié à l'historique de la relation entre les partenaires) et normatif (fondé sur le partage de normes, de valeurs ou de croyances) (DAVOINE et al. 2000, p. 69).

Ces trois canaux contribuent à inscrire la confiance dans un processus de socialisation professionnelle qui interviendrait en Allemagne dès la période d'apprentissage. Ce processus de socialisation existerait également en France, mais il jouerait moins en faveur d'une identification à l'entreprise qu' au groupe ouvrier et entraînerait plutôt une culture de conflit de classe que de coopération intra-organisationnelle. La confiance tacite serait par ailleurs favorisée par le recrutement des apprentis par les entreprises dans lesquelles ils ont été formés et par une politique paternaliste caractéristique du dense tissu de petites et moyennes entreprises allemandes (BERGHOFF, 2006;

1. Que N. LuHMANN (2006) distingue de la confiance personnelle. 
Koтthoff, 2010). Ainsi définie, la confiance tacite suggère que ce n'est pas tant le système de formation qui est producteur de confiance, que le tissu social et relationnel au sein duquel la formation s'insère et qu' elle contribue à construire.

Après une brève présentation des systèmes français et allemand de formation professionnelle initiale, nous nous arrêtons sur leurs rôles respectifs dans la production de la confiance tacite dans chacune des unités étudiées. Nous élargissons l'analyse aux modes de recrutement ouvrier, pour montrer qu'au-delà des dispositifs de formation initiale, ce sont les parcours d'entrée des salariés dans l'entreprise et d'installation dans leur premier poste à durée indéterminée qui se révèlent décisifs dans la production d'un écosystème plus ou moins propice à la confiance intra-organisationnelle.

\section{Formation professionnelle et apprentissage en alternance}

À travers des parcours d'apprentissage en alternance, le système « dual » allemand de formation professionnelle associe fortement les entreprises à la formation initiale des ouvriers (LASSERRE, 2014). En France, la relation entre formation professionnelle initiale et monde de l'entreprise est plus lâche. Dernière tentative en date pour remédier à ces liens distendus, la loi 2018 « Pour la liberté de choisir son avenir professionnel » fait basculer la régulation de l'apprentissage du domaine de la formation initiale vers celui de la formation continue auquel sont associés les partenaires sociaux. Pour autant, le système français reste «dualiste », caractérisé par la coexistence de deux statuts différents, scolaire ou professionnel, de l'apprentissage et des apprentis (DAYAN, 2018). Unifié, le système « dual » allemand repose sur le principe de l'alternance entre école professionnelle (Berufsschule) et travail en entreprise, tandis qu'en France, coexistent trois filières différentes de formation professionnelle: les lycées professionnels sous tutelle de l'Éducation nationale ; l'apprentissage en alternance entre l'entreprise et des centres de formation d'apprentis (CFA) dont la création s'appuie sur des conventions entre les régions et d'autres partenaires; le contrat de professionnalisation qui, en 2004, fait suite au contrat de qualification instauré par les partenaires sociaux en 1983 (SANCHEZ, ZAMORA, 2007). Les deux premières filières préparent aux mêmes diplômes (certificat d'aptitude professionnelle [CAP], brevet d'études professionnelles [BEP], baccalauréat professionnel et brevet professionnel), mais les élèves des lycées réalisent leur formation sous statut scolaire, alors que les apprentis sont sous statut salarié, à l'instar des jeunes en contrat de professionnalisation.

Généralisé en Allemagne, ce statut salarié facilite le tissage de liens fondés sur l'intégration à un collectif de travail sur toute la durée de la formation. C'est pourquoi certains auteurs voient dans l'alternance un facteur favorable au développement de la confiance intra-organisationnelle (DAVOINE et al., 2000) ou soulignent l'indéniable atout sociétal de la confiance ainsi faite aux jeunes en leur permettant très tôt d'intégrer le monde du travail (DAYAN, 2018). 
En France, les jeunes préparant un diplôme professionnel de premier niveau se trouvent majoritairement dans les lycées : 665190 en 2016 contre 405205 en apprentissage dans les CFA à comparer aux 1321197 apprentis allemands la même année ${ }^{2}$. La structure dualiste française reste ainsi dominée par ce qu'É. VERDIER (2017) appelle le « régime académique » de la formation professionnelle par contraste avec le « régime corporatiste », marque de fabrique du système allemand. Mais celui-ci tend aussi à évoluer, sous les effets conjugués d'un manque de places d'apprentissage en entreprises et d'une baisse d'attractivité du système dual qui, depuis 2013, recrute moins que l'université (Autorengruppe BerichterstatTung, 2018; Granato, Moreau, 2019). É. VERDIER, mais aussi Justin J. W. Powell et ses co-auteurs (2012) décrivent plus largement une hybridation croissante des systèmes français et allemands de formation professionnelle entre différents régimes d'action publique.

\section{Travail intérimaire et formation en alternance: deux modes d'entrée chez OilGum}

Tous les ouvriers de notre échantillon sont entrés chez OilGum FR par une mission d'intérim, à l'exception de deux ouvriers aujourd'hui âgés, embauchés d'abord en contrat à durée déterminée (CDD). Au-delà du passage obligé par l'intérim, on constate un effet de génération: tandis que les ouvriers plus âgés ont quitté l'école vers 16 ans, la génération des 20-35 ans est en général dotée d'un baccalauréat professionnel ou technologique. En revanche, seulement deux des seize ouvriers interrogés disposent d'une formation initiale en chimie, les autres sont boulangers, menuisiers, électriciens, fraiseurs ou outilleurs de formation et ont pour la plupart exercé ces métiers avant d'intégrer OilGum FR. Certains d'entre eux évoquent des parcours discontinus - une reconversion, un apprentissage avorté, un licenciement, etc. - qui les ont amenés à se repositionner sur le marché du travail ; d'autres ont cherché un travail qui leur convenait mieux que celui qu'ils occupaient, moins pénible, plus proche du domicile, mieux rémunéré ou avec une plus grande sécurité de l'emploi.

À leur arrivée, presque tous les ouvriers sont affectés au poste de conditionnement du secteur finition, où ils doivent surveiller l'emballage du caoutchouc, son déchargement automatique et, en cas de panne, décharger eux-mêmes la ligne. C'est le poste dont le coefficient de rémunération est le plus bas et le travail jugé le moins intéressant par les salariés. Une fois en CDI, les ouvriers peuvent ensuite évoluer vers d'autres postes. La règle de l'ancienneté régit la mobilité interne: tous les ouvriers doivent « commencer en bas » et attendre leur tour pour progresser ${ }^{3}$. Un seul ouvrier de notre échantillon, doté d'un baccalauréat en chimie et d'une expérience préalable dans le domaine, est entré directement dans le laboratoire de contrôle-qualité. Le second titulaire d'un baccalauréat en chimie n'avait quant à lui pas d'expérience préalable, et

2. Direction de L'Évaluation, De la Prospective et De la Performance, 2018, p. 93 et 137; Bundesinstitut FÜr BERUFSBILDUNG, 2018, p. 102.

3. Voir plus généralement sur ce point MisseT, 2013. 
nous raconte qu'il a dû travailler plusieurs années au poste de conditionnement avant d'évoluer. Ces exemples montrent le poids attribué à l'expérience dans le métier, quels que soient le niveau et la spécialité du diplôme initial.

À rebours de cette voie d'accès par l'intérim et d'évolution par l'ancienneté sans nécessaire qualification initiale dans le métier, la figure de l'ouvrier qualifié (Chemikant), passé par le système dual de formation professionnelle, domine chez OilGum DE. Le diplôme professionnel est ici central : ceux qui n'en disposent pas voient leur avancement bloqué, alors que le fait de le détenir stabilise les positions et permet d'éventuelles promotions. Sur les dix-huit ouvriers interrogés, quatorze ont fait leur apprentissage dans le centre de formation du Chempark ${ }^{5}$. Au terme de leur apprentissage, si les circonstances démographiques et économiques sont favorables à l'embauche, les apprentis peuvent prétendre à un large éventail de postes, sans passage obligé par le conditionnement comme dans le cas français et sans faire de preuves supplémentaires. L'intérim comme voie d'accès est minoritaire, bien que présent : quatre ouvriers sont entrés par cette voie ${ }^{6}$.

Dominés par le système dual, les parcours d'entrée et d'installation chez OilGum DE sont propices à une socialisation précoce, susceptible d'alimenter la dimension relationnelle de la confiance tacite. Pendant leur période d'apprentissage, les jeunes ont le temps de se familiariser avec le travail, comme l'exprime Ümit, ouvrier de production, 49 ans, trente-trois ans d'ancienneté: « J'ai fait beaucoup d'erreurs au début, mais on ne m'en a pas voulu, jusqu'à ce que j' aie tout appris. J'étais bien accueilli, il n'y avait pas de souci ». Effectuant des stages dans diverses unités, les apprentis ont également le temps de nouer des relations avec différents collègues. Le Betriebsrat (conseil d'entreprise) ${ }^{7}$ accompagne activement ces parcours qui permettent aux apprentis de tisser des relations pouvant ensuite être réactivées lorsqu'ils seront en poste ${ }^{8}$.

Sur le site français, la confiance ne se construit pas dans un parcours d'apprentissage, mais sur le poste. C'est même la condition sine qua non pour entrer dans le collectif ouvrier. Les récits recueillis montrent qu'il s'agit d'une confiance qui se rapporte au travail concret et aux équipes de travail, dont il faut apprendre les règles implicites afin d'instaurer des relations de confiance. Les nouveaux doivent faire leurs preuves, accepter les hiérarchies internes et attendre leur tour pour la cédéisation et l'évolution de carrière.

4. De fait, le seul ouvrier sans diplôme professionnel rencontré travaille au conditionnement depuis son embauche.

5. Sur les quatre ouvriers qui n'ont pas été formés en interne, un a appris le métier de Chemikant dans une autre entreprise, deux sont passés par d'autres métiers et un seul n'a pas de diplôme. Sur les dix-huit ouvriers rencontrés, seulement deux des ouvriers sont titulaires d'un baccalauréat.

6. Dont deux sans qualification dans l'industrie chimique (mais diplômés d'un autre métier). Sur le développement de l'accès au CDI par l'intérim en Allemagne, voir LinNe, Vogel, 2003.

7. Le conseil d'entreprise allemand se caractérise par un domaine d'intervention et un pouvoir d'action nettement plus étendus que ceux du comité social et économique français (comité d'entreprise au moment de l'enquête). Nous reviendrons en détail sur ce point dans la partie suivante.

8. La proximité relationnelle est en outre alimentée par des pratiques historiques de recrutement familial chez OilGum DE, alors qu'OilGum FR s'oppose à l'embauche familiale en dépit des demandes ouvrières. 
Pour conclure, nous retiendrons que les ouvriers du site français entrent plus tard dans l'entreprise, souvent après une expérience dans d'autres secteurs et commencent au bas de l'échelle avant d'être formés à d'autres postes, comme c'est souvent le cas dans l'industrie chimique (LegaY, SÉchaud, 2017). Chez OilGum DE, c'est par l'apprentissage que la plupart des ouvriers accèdent à l'embauche. Cette situation concerne aujourd'hui un quart seulement des apprentis allemands ${ }^{9}$, mais elle est plus répandue dans la chimie où $55,2 \%$ des apprentis décrochent un CDI au terme de leur apprentissage (en 2018) ${ }^{10}$. OilGum DE est de ce point de vue typique des modes d'accès au marché du travail ouvrier par le système dual et des leviers de production de la confiance qui lui sont associés.

\section{Similarité des canaux de recrutement chez Flybest}

Contrairement à OilGum, les voies d'accès au poste d'ouvrier en CDI sont chez Flybest relativement proches d'une unité à l'autre. Dans l'unité allemande, sur les quinze ouvriers interviewés, tous sont sans surprise passés par l'apprentissage. Mais seulement sept d'entre eux l'ont effectué chez Flybest ${ }^{11}$. Les huit autres, âgés de 30 à 56 ans, sont dotés d'une expérience professionnelle dans d'autres entreprises. Plus que dans la chimie, ce cas de figure est fréquent et ancien dans la métallurgie où les ouvriers peuvent apprendre le métier dans des petites entreprises avant de s'orienter vers la grande industrie où les emplois sont réputés plus stables et mieux rémunérés (BRZINSKY-FAY et al., 2016). Ce qui est davantage significatif d'un changement de modèle par rapport aux canons du passé, c'est que trois seulement de ces personnes ont été recrutées sur la base d'un contrat avec période d'essai contre quatre à la suite d'un contrat intérimaire. Ces exemples montrent que le diplôme d'ouvrier qualifié ne protège plus contre l'intérim et la précarité de l'emploi, notamment lorsque l'entreprise où la formation a été suivie ne recrute pas et n'a pas une réputation suffisamment solide pour faciliter l'embauche ailleurs, ou lorsque le détenteur du diplôme effectue une mobilité, voire une reconversion professionnelle.

L'unité française contribue encore davantage à brouiller les canons établis de la comparaison franco-allemande. Sur les quinze ouvriers interviewés, six sont en effet passés par une formation professionnelle en alternance auprès de Flybest avant d'être recrutés une fois le diplôme obtenu. Âgés de 20 à 29 ans, ils figurent, comme en Allemagne, parmi les plus jeunes de l'unité. Flybest est l'une des rares entreprises françaises dotée de son propre lycée professionnel. Sous statut de droit privé, il prépare les élèves sélectionnés aux métiers de l'aéronautique par la voie

9. Christian BRZINSKY-FAY et ses co-auteurs (2016) montrent que la taille de l'entreprise est un facteur important pour le passage immédiat de l'apprentissage au CDI dans la même entreprise.

10. Voir la Ausbildungsbilanz (le bilan de la formation professionnelle initiale) de la IGBCE, le syndicat industriel du secteur des mines, de la chimie et de l'énergie qui est le troisième plus grand syndicat allemand (BIRKE, DRIBBUSCH, 2019). https://2019.igbce.de/xxii-41-ausbildungsbilanz-2018/177248; consulté le 20 novembre 2020.

11. Âgés de 21 à 29 ans, ils comptent parmi les ouvriers cédéisés les plus jeunes de l'unité. 
de l'alternance. Atypique par rapport aux pratiques des lycées publics, il témoigne d'une initiative pionnière d'hybridation entre modèles académique et corporatiste de la formation professionnelle. Sur les six anciens ouvriers du lycée que nous avons interviewés, cinq sont titulaires d'un CAP et un, le plus jeune, d'un baccalauréat. La disparition en 2013 du CAP Électricien de la liste des diplômes préparés par le lycée atteste de l'élévation des prérequis de recrutement des ouvriers de l'unité électrique, désormais embauchés au niveau bac professionnel lorsqu'ils sortent de la formation professionnelle en alternance.

Deux autres canaux d'accès au CDI ouvrier existent dans l'unité française : le contrat de qualification et l'intérim. À partir du milieu des années 2000 toutefois, ce dernier s'impose comme principal canal de recrutement en dehors de la formation initiale en alternance ${ }^{12}$. Ce fut le cas pour six des quinze ouvriers rencontrés. Cédéisés après une période d'intérim plus ou moins longue - entre six et dix-huit mois-, ils sont pour trois d'entre eux diplômés avec une expérience professionnelle préalable - deux détiennent un CAP, le troisième un baccalauréat - et, pour les trois autres, sans diplôme mais dotés d'une expérience professionnelle. C'est là une différence avec l'unité allemande où tous les ouvriers interviewés sont titulaires d'un diplôme professionnel, fussent-ils ou non passés par l'intérim.

La formation initiale en alternance s'avère ainsi côté français un facteur d'intégration à l'entreprise et de développement d'une confiance tacite chez les jeunes recrues comparable à celle que l'on observe côté allemand. On relève dans les deux unités les mécanismes réputationnels, relationnels et normatifs décrits par É. DAVOINE et ses co-auteurs (2000) pour l'Allemagne des années 1990. Ainsi, Valentin souligne, dans l'extrait suivant, la relation de confiance qu'il a construite avec le tuteur ${ }^{13}$ qui l'a accompagné pendant son apprentissage et ses premiers mois en poste:

«Enquêteur: Vous avez confiance dans l'écoute de votre chef?

- Oui, ça fait longtemps que je le connais, ça a été mon tuteur sur le poste où j'ai commencé, donc ça fait longtemps que je le connais, il n'y a pas de problème sur ça. »

(Entretien réalisé avec Valentin, ouvrier, 29 ans, compagnon, douze ans d'ancienneté)

Le tutorat vaut d'ailleurs pour l'ensemble des nouvelles recrues quels que soient leur âge, leur canal de recrutement et leur expérience professionnelle préalable, et cela dans les deux unités. Ainsi, à leur arrivée, les intérimaires effectuent un stage de formation d'une durée minimale de deux semaines avant d'être affectés à un poste, sur lequel ils sont encadrés par un tuteur qui travaille à leurs côtés. En raison de règles de sécurité drastiques dans le secteur aéronautique, le personnel fraîchement arrivé sur la ligne n'est pleinement opérationnel et autonome sur son poste qu'après six mois de formation et de tutorat. Quel que soit le type de contrat, le tutorat contribue à la socialisation à l'entreprise (MOREAU, 2016), à travers le développement de relations

12. En 2017, $96 \%$ des embauches en contrats temporaires dans le secteur industriel correspondaient à des contrats d'intérim (MiLin, 2018).

13. Ouvrier expérimenté, il a suivi une formation au tutorat et travaille lui-même sur la ligne. 
interpersonnelles, mais aussi par la transmission de valeurs relatives à la manière de travailler, tant du point de vue des normes techniques et des règles de sécurité que des attendus en matière d'autonomie, d'implication et de participation des ouvriers. Le tuteur est un acteur clé de ce processus de socialisation et d'intégration.

Si notre enquête montre que les mécanismes de production de la confiance tacite par la formation initiale peuvent être effectifs également dans l'entreprise française lorsqu'existent des conditions similaires de formation en alternance à celles de l'entreprise allemande, elle révèle par ailleurs que ces mécanismes ne concernent qu'une partie des recrutements côté allemand. Elle établit enfin que certains médiateurs de la confiance caractéristiques de la formation professionnelle en alternance, tels que le tutorat, peuvent s'avérer opérants pour d'autres canaux de recrutement, dont l'intérim. On peut toutefois formuler l'hypothèse que l'absence de confiance contractuelle est dans ce dernier cas susceptible de miner la confiance tacite ainsi produite, tout du moins de la fragiliser, en particulier dans les entreprises où les possibilités de cédéisation sont statistiquement faibles.

\section{Promouvoir la confiance intra-organisationnelle par la codétermination? Du « devoir » de confiance à sa mise en œuvre}

La codétermination est un autre facteur de différenciation des mondes français et allemand de l'entreprise. Elle s'avère d'autant plus pertinente pour notre propos que le droit allemand l'assoit sur un « devoir » de confiance réciproque entre l'employeur et les représentants des salariés, faisant ainsi de la confiance un principe juridique constitutif de l'entreprise conformément à la loi constitutionnelle de l'entreprise (Betriebsverfassungsgesetz ${ }^{14}$, BetrVG par la suite). Bien davantage que la formation professionnelle par alternance, la codétermination reste un élément de confiance contractuelle sans équivalent en France.

Nous revenons dans un premier temps sur la codétermination et son assimilation à un dispositif de « coopération dans la confiance » par le droit constitutionnel allemand de l'entreprise ( $\$ 2$ Abs. 1, BetrVG). Nous comparons les caractéristiques et les compétences de son organe principal, le Betriebsrat, à celles du comité d'entreprise français transformé par l'ordonnance du 22 septembre 2017 en comité social et économique $^{15}$. Nous soumettons ensuite ces cadres institutionnels à l'épreuve de la situation d'incertitude que traversent OilGum et Flybest au moment de l'enquête. Si le cas de

\footnotetext{
14. Pour la dernière version 2020, https://www.gesetze-im-internet.de/betrvg/BetrVG.pdf; consulté le 13 novembre 2020 .

15. Ordonnance $n^{\circ} 2017-1386$ du 22 septembre 2017 relative à la nouvelle organisation du dialogue social et économique dans l'entreprise et favorisant l'exercice et la valorisation des responsabilités syndicales. Nous garderons toutefois dans cet article l'expression « comité d'entreprise » en usage au moment de l'enquête.
} 
OilGum tend à conforter dans ses grandes lignes les attendus de la comparaison francoallemande, celui de Flybest révèle comment des formes de confiance tacite construites dans l'activité même de travail peuvent, côté français, venir compenser l'absence de confiance contractuelle liée aux modes de représentation du personnel et comment, à l'inverse, les médiations institutionnelles de la confiance contractuelle peuvent, côté allemand, se trouver remises en question par des dynamiques organisationnelles.

\section{La confiance mutuelle entre employeur et représentants des salariés : un « devoir » constitutif du droit allemand de l'entreprise}

Hérités de la République de Weimar, les Betriebsräte ont vu leur périmètre redéfini par la loi constitutionnelle de l'entreprise (Betriebsverfassungsgesetz) de 1952. Les principes de la codétermination (Mitbestimmung), dont le Betriebsrat est un acteur central, s'y trouvent étroitement articulés à l'instauration d'un syndicat unique et à l'affirmation de l'autonomie des partenaires sociaux dans la négociation collective. Régulièrement actualisé, le droit constitutionnel de l'entreprise n'a pas d'équivalent en France où cette dernière demeure un objet juridique non identifié, comme le soulignent les appels à une «refondation de l'entreprise » (Hatchuel, SEgrestin, 2012; FAVEREAU, 2016).

En Allemagne, le collectif des salariés (Belegschaft) est doté d'une reconnaissance juridique, il élit ses représentants qui siègent au sein du Betriebsrat, partenaire de négociation de l'employeur. Le Betriebsrat se distingue du comité d'entreprise français sur plusieurs points. Il est composé uniquement de représentants élus des salariés, qui désignent en leur sein leur président, alors que le comité français est présidé par l'employeur. Autre différence, le Betriebsrat dispose de droits de codétermination dont ne peut se prévaloir le comité d'entreprise français qui est simplement « informé et consulté » (Code du travail, article L. 2312-8) ${ }^{16}$. Enfin, il a une fonction de défense des salariés en cas de licenciement, ce que n'a pas le comité d'entreprise.

Les salariés sont ainsi dotés d'une capacité collective d'agir dans l'entreprise par l'entremise du Betriebsrat. Celui-ci est en contrepartie soumis à un devoir de «paix sociale ( Friedenspflicht) et de « coopération dans la confiance» (Vertrauensvolle Zusammenarbeit) (§2 Abs. 1, BetrVG). Il est juridiquement enjoint à ne pas alimenter ni soutenir d'agitation contre l'employeur et à prendre en compte l'intérêt de l'entreprise au même titre que celui des salariés. La notion de confiance se trouve ici étroitement associée à l'idée de coopération en vue du bien commun des salariés et de l'entreprise (zum Wohl der Arbeitnehmer und des Betriebs). Elle appelle à transcender les divergences entre les parties prenantes et à encourager la recherche de solutions

16. Les sujets soumis à codétermination sont énumérés par la loi (NIEDENHOFF, 2005) : règlement intérieur de l'entreprise, temps de travail, rémunérations, congés et plannings, évaluation des salariés, prévention des accidents du travail, promotion de la santé au travail et protection du travail, travail en équipes autorégulées. Pour une comparaison entre la consultation à la française et la codétermination à l'allemande, voir Thierry NoBRE, 2000. 
négociées. L'objectif du droit du travail est alors d'équiper les personnes et les collectifs pour qu'ils puissent s'engager dans la construction d'une relation de confiance.

Un autre dispositif, sans fondement légal cette fois, vient soutenir la production de la confiance dans l'entreprise allemande. Il s'agit des «personnes de confiance » (Vertrauensleute), qui sont des hommes et des femmes proches du syndicat qui interviennent au quotidien dans les équipes comme des facilitateurs de communication entre les ouvriers, l'encadrement et le Betriebsrat. Ils se distinguent des délégués syndicaux français d'abord parce qu'ils sont élus par les seuls salariés syndiqués, ensuite parce qu'ils ne bénéficient d'aucun crédit d'heures et d'aucun statut légal. Leur présence est forte dans les branches de la chimie et de la métallurgie dont relèvent nos deux entreprises.

Betriebsrat et Vertrauensleute font partie du modèle allemand de «partenariat conflictuel » (MüLLER-JENTSCH, 1999) qui cherche toutefois aujourd'hui un second souffle (KÄDTLER, 2012). Son érosion se manifeste notamment par la baisse du taux de syndicalisation des salariés, du nombre d'entreprises adhérant aux conventions collectives et du nombre de salariés travaillant dans une entreprise où existe un Betriebsrat ${ }^{17}$.

\section{OilGum: coopération dans la confiance et capacité d'agir du Betriebsrat}

OilGum DE incarne de manière typique la «coopération dans la confiance » prônée par le droit allemand que nous venons de décrire. L'entreprise s'inscrit dans le modèle de « partenariat de la chimie » (Chemiepartnerschaft), caractérisé par une continuité des relations de coopération entre partenaires sociaux au niveau de la branche et des entreprises (KÄDTLER, 2006). Le Betriebsrat, composé de membres du syndicat IGBCE, travaille main dans la main avec la direction des ressources humaines (DRH). Il est très investi dans le recrutement des nouveaux ouvriers et apprentis, domaine qui, en France, est sous le contrôle étroit du département RH et des responsables d'ateliers. Il accompagne les parcours d'intégration des apprentis et s'assure de la construction de leur expérience par leur passage dans différentes unités. On constate par ailleurs une grande proximité du Betriebsrat avec les «personnes de confiance » de l'entreprise, de même qu'avec l'encadrement de proximité. L'ancienne directrice du Betriebsrat d'OilGum DE que nous avons rencontrée (66 ans, quarante-sept ans d'ancienneté), Bettina, nous explique qu'elle s'est même souvent adressée à l'encadrement pour que celui-ci encourage les salariés à se présenter aux élections: "On parle avec les supérieurs et on leur dit: "Allez, faites un peu de publicité !" » Plusieurs membres du Betriebsrat occupent d'ailleurs ou ont occupé des positions d'encadrement ${ }^{18}$.

\footnotetext{
17. Le principe de l'extension des conventions collectives qui prennent force de droit pour toutes les entreprises du secteur ne s'applique pas en Allemagne. Sur ces transformations, voir la controverse autour de la notion de Konflitkpartnerschaft dans INDUSTRIELLE BEZIEHUNGEN - ZEITSCHRIFT FÜr ARBEIT, ORGANISATION UND MANAGEMENT, 2016, $\mathrm{n}^{\text {os }} 1$ et 3 ; voir également: https://www.iab-forum.de/die-betriebliche-mitbestimmung-verliert-an-boden/; consulté le 20 novembre 2020.

18. Son président et vice-président au moment de l'enquête sont d'anciens contremaîtres. L'un d'eux est même devenu contremaître alors qu'il était déjà engagé dans le Betriebsrat.
} 
Ces relations de coopération tissées au sein de l'organisation ont favorisé l'action du Betriebsrat à des moments clés de l'histoire de l'entreprise. Il a ainsi pu éviter l'externalisation du secteur de la maintenance grâce aux bonnes relations avec les managers qui, très tôt, lui ont donné des informations sur le projet; lors du détachement de TopGum de l'entreprise initiale Hoffmann (encadré 2), il a obtenu un vote favorable du personnel concernant une réduction du temps de travail de cinq heures par semaine, ce qui a permis de réduire le nombre de licenciements prévus. En outre, lors d'un programme de restructuration au milieu des années 2010, les Betriebsräte des différents sites de l'entreprise se sont entendus - non sans conflits internes - pour négocier avec succès la répartition équitable des licenciements sur les trois sites lors d'un conseil d'administration. Enfin, lors de la création du joint venture, le Betriebsrat a pu éviter des licenciements et assurer le maintien des avantages sociaux.

Sur le site français d'OilGum, les relations entre représentants du personnel et encadrement sont d'une autre nature. Comme souvent en France, les rôles de représentant du personnel et de manager sont difficilement conciliables (GIRAUD, 2015; AMOSSÉ, DENIS, 2016). La plupart des représentants nous racontent que leur salaire a été « gelé » depuis qu'ils exercent leur mandat, alors que la responsable RH (Aurélie, 45 ans, deux ans d'ancienneté) nous fait part de sa stratégie pour attiser la division syndicale: « On sait que les syndicats se font aussi des guerres entre eux sur certains sujets, donc en fonction des sujets, on va d'abord s'adresser à un syndicat avant un autre. » Le paysage syndical, longtemps dominé par la Confédération française démocratique du travail (CFDT) avant que celle-ci soit détrônée aux dernières élections par la Confédération française du travail chrétien (CFTC), est de fait marqué par la concurrence et la division ${ }^{19}$. Chez OilGum, dans ce contexte de relations professionnelles très contrastées d'un site à l'autre, la grande majorité des salariés allemands interrogés déclarent faire confiance au Betriebsrat et le considèrent même comme un acteur central du fonctionnement de l'entreprise, tandis que les salariés français ont des avis beaucoup plus réservés quant au rôle joué par les instances représentatives du personnel.

\section{Flybest: la confiance en berne entre les salariés et le Betriebsrat}

Chez Flybest, la montée en charge continue d'une ligne de production et la baisse d'une autre, chacune dédiée à un modèle spécifique d'avion, engendrent sur les deux sites d'importants mouvements de personnels couplés à une réorganisation du travail. Si des tensions existent, elles ne sont cependant pas source d'inquiétude dans l'unité française et ne dégénèrent pas en crise. La situation est différente chez Flybest DE où la montée en charge a créé un retard de livraison de dix-sept avions. La pression qui en résulte, notamment en ce qui concerne les heures supplémentaires, est concomitante du renvoi simultané de nombreux travailleurs intérimaires (Leiharbeitskräfte ou LAK).

19. La CFDT a été accusée par les autres syndicats (CFTC, FO [Force ouvrière], CFE-CGC [Confédération française de l'encadrement - Confédération générale des cadres] et CGT [Confédération générale du travail]) de favoriser les augmentations « à la tête du client » et de jouer le jeu de la direction. 
Il s'agissait pour l'entreprise d'anticiper la mise en conformité avec une loi que le Bundestag devait adopter ${ }^{20}$ et qui réduit la durée du travail intérimaire au sein d'une même entreprise à dix-huit mois (trente-six par dérogation dans des secteurs comme celui de Flybest soumis à des normes particulières de sécurité). Or de nombreux travailleurs temporaires étaient en poste chez Flybest DE depuis plus de dix, voire quinze ans, et ce de manière parfaitement légale. La situation crée beaucoup d'émoi dans les équipes dont les intérimaires présents de longue date étaient devenus des membres à part entière. Elle est également source d'incompréhension: pourquoi se séparer de ces travailleurs expérimentés plutôt que de les embaucher, alors que l'usine n'arrive pas à assurer les livraisons et fonctionne en heures supplémentaires ?

«Liquidés... les gens sont partis, juste partis. Maintenant, c'est clair, on nous demande de travailler les week-ends, c'est clair, toujours le samedi, le dimanche, pour rattraper un peu de retard et revenir un peu dans le jeu. Là, il faut vraiment se demander comment quelque chose comme ça peut arriver, comment des décisions aussi gravissimes sont prises. [...] Tout en bas, à la base, on essaye vraiment quotidiennement d'accomplir notre devoir, de faire notre travail, et des erreurs comme ça sont commises...»

(Sebastian, ouvrier, 44 ans, seize ans d'ancienneté)

De la combinaison de ces deux facteurs conjoncturels - le rééquilibrage des lignes de production et la mise en conformité avec la nouvelle loi sur le travail temporaire - naît une situation de forte tension qui entame, chez les ouvriers interrogés, la confiance dans la direction du site allemand, mais également dans son Betriebsrat dont le mutisme et le consentement à cette politique sont critiqués avec amertume et déception. Les salariés rencontrés accusent ce dernier de jouer le jeu de la direction plutôt que de défendre leurs intérêts. Attestant de la profondeur du malaise, deux d'entre eux, dont un élu syndical, ont rendu leur carte au syndicat IG Metall quelques jours avant l'entretien.

La confiance dans les instances de représentation du personnel n'est pas davantage au rendez-vous chez Flybest FR, mais il s'agit là d'un résultat plus attendu. Force ouvrière (FO), la CGT et la Confédération générale des cadres (CGC) sont représentées dans l'unité. FO est largement majoritaire. Ses délégués définissent d'abord leur mission comme un rôle d'accompagnement des salariés dans la formulation de demandes individuelles variées (mobilité, aménagement du temps de travail, salaires, etc.), mais aussi, et de manière inattendue, de coopération constructive avec l'employeur:

«C'est un syndicat qui amène du plus aux salariés mais sans mettre en péril l'entreprise. [...] Et c'est un truc que j' ai trouvé super, parce que voilà tout travail mérite salaire, les conditions de travail, c'est important aussi pour les salariés, mais il faut que l'usine vive quoi. Et donc je trouvais qu'il y avait de bons compromis, toujours des accords gagnant-gagnant. »

(Julien, compagnon et délégué FO, 37 ans, quinze ans d'ancienneté)

20. Elle fut votée le 21 octobre 2016. 
Le délégué FO place d'ailleurs ses rapports avec la direction et l'encadrement sous le sceau de la confiance. De son côté, la responsable RH souligne le rôle de médiateur des délégués, surtout de ceux de FO, dans le suivi des demandes individuelles. À une exception près, les chefs d'équipe et cadres partagent ce sentiment, que l'on associe de prime abord plutôt au fonctionnement allemand, de coopération dans la confiance entre encadrement et délégués du personnel. Adolfo (chef d'équipe, 33 ans, treize ans d'ancienneté) s'est fait des délégués de l'unité « plutôt des alliés »; Thomas (cadre, responsable du service qualité et planification, 39 ans, quatorze ans d'ancienneté) dit être « en communication » avec eux tout en soulignant le caractère « atypique » de ce genre de rapports, tout particulièrement dans un moment de profonde réorganisation de deux lignes de production, l'une d'entre elles étant désormais appelée à fonctionner, selon le modèle de l'entreprise libérée, en équipe autorégulée sans chef d'équipe. De manière générale, l'unité française est peu sujette aux épreuves de force entre direction et délégués syndicaux; celles-ci se jouent à d'autres niveaux du site et de l'entité nationale.

Pour autant, l'action des délégués du personnel et leurs relations avec le personnel d'encadrement n'interviennent pas dans les récits des salariés de Flybest FR sur la production de la confiance. Ce sont d'autres appuis qui sont conviés comme creusets de la confiance organisationnelle: les pratiques de management participatif et le degré d'autonomie dans le travail, mais aussi le développement professionnel, au sens d'évolution dans le poste ou la carrière et les possibilités de sortie de la condition ouvrière.

Pour résumer, alors que le cas de OilGum établit la persistance de médiations institutionnelles de la confiance côté allemand, l'exemple de Flybest met en lumière les dynamiques organisationnelles qui affectent la confiance; dans ce cas précis, négativement côté allemand, positivement côté français.

\section{Le poids de la position des unités dans le groupe}

Comme le souligne Reinhard BACHMANN (2003), la coopération organisationnelle se déploie dans une combinaison de relations de confiance et de pouvoir. La riche veine de travaux sur les entreprises multinationales montre combien les relations entre siège et filiales sont le théâtre d'intenses luttes de pouvoir autour de l'allocation des ressources, de la définition des politiques de l'entreprise et du transfert de pratiques organisationnelles (MAYRHOFER, 2011; FERNER et al., 2012). Certaines recherches révèlent en outre l'importance des configurations locales des filiales, des jeux micro-politiques qui s'y déploient et qui offrent aux acteurs locaux des marges de manœuvre face aux politiques du groupe multinational (GEPPERT, DÖRRENBÄCHER, 2014). Cependant, même lorsqu'ils adoptent une perspective par le bas, ces travaux se focalisent sur le management et les représentants du personnel et s'intéressent peu à la façon dont les salariés évaluent les relations de pouvoir au sein de l'entreprise. Or nos entretiens montrent que la manière dont les salariés perçoivent ces relations contribue 
à façonner leur confiance systémique dans l'entreprise. Ainsi les salariés interprètent l'instabilité organisationnelle d'OilGum et la politique de Flybest comme les signes avant-coureurs d'une modification des rapports de force entre les différentes unités de l'entreprise. Ces interprétations et la façon dont elles affectent la confiance des salariés sont intimement liées à l'histoire respective des unités et à leur position vis-à-vis des centres de décision de la multinationale.

\section{OilGum: la proximité historique entre l'unité de production et le siège comme marqueur identitaire}

En dépit de la relative conformité d'OilGum aux attendus de la comparaison franco-allemande en matière de formation et de relations professionnelles, la création du joint venture a davantage affecté la confiance dans l'entreprise des salariés allemands que des salariés français.

«Le changement vers OilGum, c'est un peu, on s'inquiète... Parce que, en quelque sorte, le... [indication d'une nationalité étrangère, supprimée pour préserver l'anonymat de l'entreprise] , il vient, il achète ça. [...] Mais, comment est-ce qu'il veut faire du profit, avec ce que TopGum avait déjà liquidé parce que ça ne générait pas assez de profit? Qu'est-ce qu'il fera autrement, sinon des économies ? [...] Pour l'instant, tout est... personne ne sait où ça nous mènera. »

(Jan, ouvrier, 32 ans, dix-sept ans d'ancienneté)

Sur le site français, l'arrivée de BigOil est envisagée de façon plus sereine. Le joint venture est vu comme "quelque chose de sérieux », alors qu' " on était à deux doigts d'être rachetés par un fonds de pension » souligne Michel, préparateur maintenance, délégué FO, 50 ans, vingt-neuf ans d'ancienneté. Certains salariés pensent que le site sera moins exposé aux fluctuations du cours du pétrole et gagnera ainsi en stabilité. C'est le cas de Nicolas, conducteur de ligne, 34 ans, quatorze ans d'ancienneté :

«Le fait d'avoir un nouveau partenaire, ça fiabilise le truc, sachant que c'est lui qui a le pétrole en plus, ça sécurise. Donc effectivement, le point de vue ressenti par rapport à ça, c'est positif quoi. »

Enfin, les salariés de l'unité française estiment que la fusion équivaut à l'arrivée d' ' argent frais », ce qui suscite espoir et confiance, après des années difficiles de réduction des investissements dans le site. Ils font valoir que la fusion aura peu d'impact sur leur travail quotidien - l'activité concrète, les tâches à accomplir, la vie des équipes.

«Le travail reste le même, ça, non, foncièrement ça change pas grand-chose je dirais... Sinon, le chef de service, ou un truc qui peut changer, mais encore... Même pas, là, y a même pas, au niveau de la hiérarchie, y a pas de gros changements, ou rien. La direction, oui, mais après, la direction nous, on la côtoie pas, on la voit qu'occasionnellement, y a pas de lien direct avec elle. »

(Nicolas, conducteur, 34 ans, quatorze ans d'ancienneté) 
Les salariés français manifestent aussi un détachement plus important par rapport à la multinationale allemande. OilGum est la sixième enseigne placée sur le toit de l'usine depuis sa création, la plupart des ouvriers ayant connu d'autres employeurs. Les salariés allemands en revanche s'identifient fortement à la multinationale pour laquelle ils ont commencé à travailler jeunes, avec la perspective d'un emploi à vie et des avantages sociaux conséquents (salaires élevés, retraite complémentaire, etc.), comme les membres de leur famille avant eux. Ce n'est que la deuxième fois que leur entreprise change d'enseigne, sachant que le passage de Hoffmann à TopGum a été vécu de manière moins dramatique, dans la mesure où TopGum s'inscrivait à leurs yeux dans la continuité d'Hoffmann.

L'unité allemande se trouve dans un Chempark qui était alors l'un des sites les plus importants de Hoffmann, à proximité du siège. Grâce à cette proximité géographique, qui permettait des réunions régulières avec les dirigeants du siège, le Betriebsrat avait une position forte contrastant avec le rapport de force et d'extériorité défavorable dans lequel se trouvaient les organisations syndicales françaises à la même époque. Si le passage à TopGum n'a pas altéré la coopération entre direction et Betriebsrat, la peur est vive, au moment de l'enquête, y compris parmi les membres du Betriebsrat, qu'à terme le joint venture soit «avalé » par BigOil: «Là on est à 50/50, mais je ne pense pas que ça va tenir à terme », nous explique Goran, président du Betriebsrat, 38 ans, dix-neuf ans d'ancienneté. Pour la première fois, les salariés allemands se trouvent devant la perspective de devoir travailler pour une entreprise étrangère, dont les centres de décision seront potentiellement hors de portée de l'encadrement allemand, mais aussi du Betriebsrat qui jusqu'alors disposait de ressources suffisantes pour amortir le choc du démembrement de l'entreprise L'histoire et la position des deux unités dans la multinationale et leurs potentielles incidences sur les rapports de pouvoir entre direction et représentants du personnel s'avèrent, pour cet épisode, décisifs dans l'alimentation d'un climat de confiance ou de défiance. Alors que les salariés français considèrent que leur unité, qui occupait une position périphérique chez Topgum, a plus à gagner qu'à perdre du joint venture, c'est le sentiment inverse qui anime les travailleurs de l'unité allemande, jusque-là force vive au cœur du groupe.

\section{Flybest: la distance propice aux rumeurs}

Chez Flybest, la grande majorité des salariés français se montrent, eux aussi et contrairement à leurs collègues allemands, confiants dans l'entreprise et son avenir, alors que des deux côtés du Rhin, les unités sont secouées par d'importantes transformations. Les médiations institutionnelles ne sont pas d'un grand recours pour expliquer ces différences. Tandis que la socialisation par l'apprentissage, caractéristique du système allemand, vaut aussi pour l'unité française, le Betriebsrat, acteur consacré de la production de la confiance dans l'entreprise allemande, fait l'objet de la défiance de nombreux salariés. 
Au-delà de la crise de confiance dans le Betriebsrat propre à Flybest, les entretiens mettent en avant, tout comme ceux d'OilGum, la prégnance de la question de la proximité/distance au centre de décision. Cette fois, cela n'est pas la proximité du site allemand au noyau historique de la multinationale qui est en jeu, mais au contraire la distance géographique au siège, qui crée un climat propice aux rumeurs. Le soupçon que « les Français veulent nous fermer » est largement répandu parmi les ouvriers allemands, alors même que le président directeur général du groupe, dont le siège est situé en France, est allemand. Bien que dénuée de tout fondement objectif, cette menace de fermeture «par les Français » est même brandie par le directeur du site allemand pour mobiliser les troupes et les exhorter aux heures supplémentaires afin d'éponger les retards de livraisons. Mais elle exerce un effet exactement inverse: elle démobilise et démoralise les salariés qui ont l'impression que quoi qu'ils fassent, la décision est politique et indépendante du travail fourni.

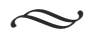

Alors que la France est volontiers caractérisée dans les comparaisons internationales par son étatisme et son centralisme (SCHMIDT, 2016), les résultats de notre enquête montrent que la confiance des salariés dans l'entreprise est davantage soutenue par des médiations institutionnelles en Allemagne qu'en France (UsunIER, 2000b). La comparaison des vagues 1999 et 2008 de l'European Value Survey révèle cependant que la confiance des Allemands dans les institutions accuse une chute entre 1999 et 2008, tandis qu'elle est en hausse en France sur la même période ${ }^{21}$. Partant de ce double constat, l'objectif de l'article était, d'une part, d'analyser l'évolution et l'actualité des médiations institutionnelles identifiées dans la littérature comparative des années 1980-1990, d'autre part, d'étudier ces médiations en action dans l'entreprise. À dessein, nous avons focalisé le propos sur deux multinationales dont les sites allemands se caractérisaient au moment de l'enquête par une remise en question de la confiance des salariés dans l'entreprise, alors que le niveau de confiance sur les sites français restait stable.

La codétermination continue de jouer un rôle important en Allemagne - tout du moins dans le secteur industriel-conformément aux missions de paix sociale et de coopération dans la confiance que lui assigne le droit de l'entreprise. Le cas de Flybest montre toutefois que l'action (ou l'inaction) de son institution phare, le Betriebsrat, peut aussi être remise en question par les salariés. La formation professionnelle, quant à elle, a connu une évolution qui tend vers la transformation, voire l'hybridation des systèmes académique et corporatiste (VERDIER, 2017). Même si les caractéristiques sociétales demeurent importantes, avec un taux de formation en alternance nettement

21. D'après les résultats de la dernière vague de 2017 (BRÉCHON et al., 2019), les taux continuent à augmenter en France pour la confiance dans le système de santé et la sécurité sociale, mais baissent pour les syndicats et le système d'enseignement. Ces taux se stabilisent en France pour les grandes entreprises. Ils remontent côté allemand, sauf pour les grandes entreprises, mais restent de 20 points en dessous des scores français, sauf pour les syndicats. 
plus élevé en Allemagne qu'en France dans le cadre d'un système dual allemand marqué par le corporatisme et un système français dualiste clivé entre filières académiques et corporatistes, l'apprentissage et le travail intérimaire sont devenus des voies de recrutement ouvrier partagées dans les deux pays. L'étude des parcours de salariés montre une diversification et une déstandardisation des voies d'accès au CDI ouvrier en Allemagne. Si le diplôme professionnel demeure incontournable, ce dernier ne protège plus contre la précarité de l'emploi induite par le travail intérimaire. Ce résultat est d'autant plus notable que l'enquête a porté sur un segment particulier du marché du travail, celui des grandes entreprises industrielles, innovantes et multinationales, dont les conditions d'emploi et de travail, mais aussi les relations professionnelles, sont globalement plus favorables aux salariés que celles du secteur des services ou des petites et moyennes entreprises.

Analyser de manière croisée les dispositifs institutionnels et leur traduction en situation dans l'entreprise conduit dès lors au constat d'un brouillage partiel des canons établis de la comparaison franco-allemande, et met en avant l'importance des contingences organisationnelles. Les relations de distance ou de proximité des unités par rapport au centre de pouvoir de la multinationale s'avèrent particulièrement déterminantes dans les cas étudiés. Ces relations peuvent alimenter ou à l'inverse entamer la confiance en fonction du contexte organisationnel, de la situation économique de l'entreprise et de l'histoire de l'unité considérée.

Cela ne signifie pas que les médiations institutionnelles nationales ne jouent plus de rôle, mais qu'elles perdent de leur pouvoir discriminant en matière de comparaison internationale. C'est particulièrement le cas des dispositifs de la formation professionnelle, alors que les institutions de relations professionnelles, bien qu'en proie à des remises en cause côté allemand, restent contrastées et structurantes, tout du moins dans le secteur des grandes entreprises industrielles que nous avons étudiées. Mener, au-delà des institutions, l'enquête sur le fonctionnement des organisations et les parcours des salariés nous confronte à une réalité complexe. L'hybridation (dans le cas de la formation professionnelle en France) ou la remise en question (dans le cas du partenariat conflictuel allemand) de ces institutions sont symptomatiques d'une recomposition plus large des héritages. Si en France, la réforme des instances du dialogue social dans l'entreprise ne s'est finalement pas orientée en 2017 vers la codétermination en dépit des discussions ouvertes dans ce sens, en Allemagne, les interventions de l'État fédéral dans la régulation des relations de travail se multiplient, en réponse à une forte segmentation du marché du travail, conséquence des lois Harz notamment, qui rend les modes de régulation négociée inopérants pour toute une frange de travailleurs (HASSEL, 2016). Si la réglementation de la durée des contrats de travail intérimaire (2016) renoue avec une législation balayée par le souffle de libéralisation des lois Hartz, la loi sur l'instauration d'un salaire minimum interprofessionnel et national (2015) marque en revanche un vrai changement de paradigme. 


\section{BIBLIOGRAPHIE}

AMossé T., DenIS J.-M. (coord.) (2016), « La discrimination syndicale en question : la situation en France », Travail et Emploi, ${ }^{\circ} 145$.

Autorengruppe Berichterstattung (2018), Bildung in Deutschland, Bielefeld.

BACHMAnN R. (2003), "Trust and Power As Means of Coordinating the Internal Relations of the Organization: A Conceptual Framework", in Nooteboom B., Six F. (eds.), The Trust Process in Organizations, Cheltenham, Edward Elgar, pp. 58-74.

Berghoff H. (2006), "The End of Family Business? The Mittelstand and German Capitalism in Transition, 1949-2000”, Business History Review, vol. 80, n 2, pp. 263-295.

Birke P., DribBusch H. (2019), Les Syndicats allemands. Défis en période de transition, Berlin, Friedrich Ebert Stiftung.

Bourrier M. (1991), Le Nucléaire à l'épreuve de l'organisation, Paris, Presses universitaires de France.

Bréchon P., Gonthier F., Astor S. (dir.) (2019), La France des valeurs. Quarante ans d'évolutions, Grenoble, Presses universitaires de Grenoble.

BRZINSKY-FAY C., EBNER C., SEIBERT H. (2016), « Veränderte Kontinuität. Berufseinstiegsverläufe von Ausbildungsabsolventen in Westdeutschland seit den 1980er Jahren », Kölner Zeitschrift für Soziologie und Sozialpsychologie, vol.68, n 2, pp. 229-258.

BUndESINSTITUT FÜR BERUfSBILDUNG (Hrsg.) (2018), Datenreport zum Berufsbildungsbericht 2018, Bonn [en ligne]: https://www.bibb.de/dokumente/pdf/bibb_datenreport_2018.pdf, consulté le 20 novembre 2020.

CARney B. M., GETZ I. (2013 [2009]), Liberté \& Cie. Quand la liberté des salariés fait le succès des entreprises, Paris, Flammarion.

DAVOINE É., WALliser B., RIERA J.-C. (2000), « La formation professionnelle initiale en France et en Allemagne: une analyse des mécanismes de confiance et de méfiance à travers deux études de cas », Revue de gestion des ressources humaines, $\mathrm{n}^{\circ} 37$, pp.57-75.

DAYAN J.-L. (2018), « La formation professionnelle des jeunes: système dual en Allemagne, système dualiste en France », Droit social, n 12, pp. 1032-1036.

DiRECTION DE L'ÉVALUATION, DE LA PROSPECTIVE ET DE LA PERFORMANCE (2018), Repères et références statistiques. Enseignements, Formation, Recherche, Paris, Ministère de l'Éducation nationale, Ministère de l'Enseignement supérieur, de la Recherche et de l'Innovation. En ligne : https://cache.media.education.gouv.fr/file/RERS_2018/28/7/depp-2018-RERS-web_1075287. pdf, consulté le 20 novembre 2020.

Eymard-Duvernay F. (1989), «Conventions de qualité et formes de coordination », Revue économique, vol.40, $\mathrm{n}^{\mathrm{O}} 2$, pp. 329-360.

FAVEREAu O. (dir.) (2016), Penser le travail pour penser l'entreprise, Paris, Mines ParisTech.

Ferner A., Edwards T., TeMPel A. (2012), "Power, Institutions and the Cross-National Transfer of Employment Practices in Multinationals", Human Relations, vol. 65, n 2, pp. 163-187. 
Les médiations institutionnelles de la confiance en entreprise. Une comparaison franco-allemande

Fukuyama F. (1995), Trust: The Social Virtues and the Creation of Prosperity, New York, The Free Press.

GEPPERT M., DöRRENBÄCHER C. (2014), "Politics and Power within Multinational Corporations: Mainstream Studies, Emerging Critical Approaches and Suggestions for Future Research", International Journal of Management Reviews, vol. 16, n 2, pp. 26-244.

GIRAUD B. (2015), « S'arracher à sa condition d'ouvrier: de l'engagement syndical à l'encadrement intermédiaire », La Revue de l'Ires, $\mathrm{n}^{\circ}$ 81, pp. 33-58.

Granato M., Moreau G. (2019), « Introduction. Les défis de l'apprentissage en Allemagne », Formation emploi, $\mathrm{n}^{\mathrm{o}}$ 146, pp. 7-28.

HASSEl A. (2016), « Le modèle allemand en transition », Revue française des affaires sociales, $\mathrm{n}^{\mathrm{o}} 1$, pp. 143-161.

Hatchuel A., Segrestin B. (2012), Refonder l'entreprise, Paris, Seuil.

HeIDENREICH M. (2012), “The Social Embeddedness of Multinational Companies: A Literature Review", Socio-Economic Review, vol. 10, n 3, pp. 549-579.

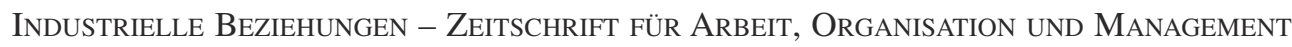
(2016), vol.23, $\mathrm{n}^{\text {os }} 1$ et 3 .

IRIBARNE P. (D') (1991), « Culture et “effet sociétal” », Revue française de sociologie, vol. 32, $\mathrm{n}^{\mathrm{o}} 4$, pp. 599-614.

IRIBARNE P. (D’) (2005), «Analyse stratégique et culture: un nécessaire retour aux sources », Revue française de sociologie, vol.46, $\mathrm{n}^{\circ}$ 1, pp. 151-170.

KÄDTLER J. (2006), Sozialpartnerschaft im Umbruch, Hamburg, VSA.

KäDTLer J. (2012), “German Social Partnership in Crisis: Proof of Value or Crisis of Social Partnership", in Deleonardis O., Negrelli S., Salais R. (eds.), Democracy and Capabilities for Voice: Welfare, Work and Public Deliberation in Europe, Bruxelles, Peter Lang, pp. 157-179.

KARPIK L. (1996), « Dispositifs de confiance et engagements crédibles », Sociologie du travail, vol. 37, $\mathrm{n}^{\circ} 4$, pp. 527-550.

Kоттноғғ H. (2010), "Betriebliche Sozialordnung" als Basis ökonomischer Leistungsfähigkeit », in Beckert J., Deutschmann C. (Hg.), Wirtschaftssoziologie, Wiesbaden, VS, pp.428-446.

LALlement M., Zimmermann B. (2019), « Tous responsables? Transformations du travail, métamorphoses de la responsabilité », Sociologie du travail [en ligne], vol.61, n 2. https:// doi.org/10.4000/sdt.17981

LASSERRE R. (2014), «La formation professionnelle en Allemagne. Dynamiques socioéconomiques et capacités d'adaptation du système dual », Regards sur l'économie allemande. Bulletin économique du CIRAC, $\mathrm{n}^{\circ} 113, \mathrm{pp} .17-32$.

LAZARIC N., LoREnZ E. (eds.) (1998), Trust and Economic Learning, Cheltenham, Edward Elgar. 
Livet P., Reynaud B. (1998), “Organizational Trust, Learning and Implicit Commitment”, in Lazaric N., Lorenz E. (eds.), Trust and Economic Learning, Cheltenham, Edward Elgar, pp. 266-284.

Legay A., SÉchaud F. (2017), «Dans les industries chimiques, “on capte, on forme, on embauche" », Céreq Bref, n ${ }^{\circ} 355$.

LinNe G., Vogel B. (Hrsg.) (2003), « Leiharbeit und befristete Beschäftigung: Neue Formen sozialer Gefährdung oder Chance auf Arbeitsmarktintegration? », Arbeitspapier, $\mathrm{n}^{\circ}$ 68, Düsseldorf, Hans-Böckler-Stiftung.

Luhmann N. (2006), La Confiance: un mécanisme de réduction de la complexité sociale, Paris, Economica.

Maurice M., Sellier F., Silvestre J.-J. (1982), Politique d'éducation et organisation industrielle en France et en Allemagne: essai d'analyse sociétale, Paris, Presses universitaires de France.

MAYRhOFER U. (dir.) (2011), «Les relations siège-filiales dans les multinationales », Revue française de gestion, $\mathrm{n}^{\mathrm{O}} 212$.

MiLIN K. (2018), «CDD, CDI : comment évoluent les embauches et les ruptures depuis 25 ans? », Dares analyses, $\mathrm{n}^{\circ} 026$.

Misset S. (2013), «Classifications ouvrières et confrontation de générations d'ouvriers qualifiés : vers une remise en cause de l'ordre de l'ancienneté? », Sociétés contemporaines, ${ }^{\circ}{ }^{90}$, pp. 29-52.

Moreau G. (2016), « L'apprenti et son maître », Recherche et formation, nº 83, pp. 19-31.

MülleR-Jentsch W. (Hg.) (1999), Konfliktpartnerschaft. Akteure und Institutionen der Industriellen Beziehungen, München und Mering, Hampp.

Niedenhoff H.-U. (2005), « Mitbestimmung im europäischen Vergleich », IW-Trends Vierteljahresschrift zur empirischen Wirtschaftsforschung, vol. 32, $\mathrm{n}^{\mathrm{o}}$ 2, pp.3-17.

Nobre T. (2000), «Une comparaison des systèmes de participation des salariés à la gestion: consultation à la française versus codétermination à l'allemande », in Usunier J.-C. (dir.), Confiance et performance. Un essai de management comparé France Allemagne, Paris, Vuibert, pp. 85-100.

PARAdeise C., Porcher P. (1990), « Le contrat ou la confiance dans la relation salariale », Travail et Emploi, $\mathrm{n}^{\mathrm{o}} 46$, pp. 5-14.

Powell J. J. W., Graf L., Bernhard N., Coutrot L., Kieffer A. (2012), “The Shifting Relationship between Vocational and Higher Education in France and Germany: Towards Convergence?", European Journal of Education, vol. 47, n 3, pp. 405-423.

SANChez R., ZAMORA P. (2007), « Retour sur quelques jalons de l'histoire de la formation professionnelle en alternance », Éducation et formations, $\mathrm{n}^{\circ} 75$, pp. 109-116.

Schmidt V. A. (2016), "Varieties of Capitalism: A Distinct French Model?", in Elgie R., Grossman E., Mazue A. G (eds.), The Oxford Handbook of French Politics, Oxford, Oxford University Press, pp. 606-635. 
Simmel G. (1999 [1908]), Sociologie. Études sur les formes de la socialisation, Paris, Presses universitaires de France.

Swidler A. (1986), "Culture in Action: Symbols and Strategies", American Sociological Review, vol.51, n 2 , pp. 237-286.

Thévenot L. (1990), «L'action qui convient», in Pharo P., Quéré L. (dir.), Les Formes de l'action, Paris, Éditions de l'EHESS, pp. 39-69.

USUNIER J.-C. (dir.) (2000a), Confiance et performance. Un essai de management comparé France Allemagne, Paris, Vuibert.

Usunier J.-C. (2000b), « Confiance, trust ou Vertrauen », in Usunier J.-C. (dir.), Confiance et performance. Un essai de management comparé France Allemagne, Paris, Vuibert, pp. 219-226.

VERDIER É. (2017), «Comment rendre compte des changements qui affectent les systèmes nationaux de formation tout au long de la vie ? Entre cohérences structurelles et hybridation », Éducation permanente, $\mathrm{n}^{\mathrm{o}} 213$, pp. 13-27.

WiLlinger M., LOHMANN C., USUNIER J.-C. (2001), « Une comparaison franco-allemande de la confiance et de la réciprocité. Une expérimentation fondée sur le jeu de l'investissement », Revue d'économie politique, vol. 111, n 1, pp.151-172. 
